\title{
Directly measuring spinal cord blood flow and spinal cord perfusion pressure via the collateral network: Correlations with changes in systemic blood pressure
}

\author{
Yuya Kise, MD, Yukio Kuniyoshi, MD, PhD, Hitoshi Inafuku, MD, PhD, Takaaki Nagano, MD, \\ Tsuneo Hirayasu, MD, PhD, and Satoshi Yamashiro, MD, PhD
}

\begin{abstract}
Objective: During thoracoabdominal surgery in which segmental arteries are sacrificed over a large area, blood supply routes from collateral networks have received attention as a means of avoiding spinal cord injury. The aim of this study was to investigate spinal cord blood supply through a collateral network by directly measuring spinal cord blood flow and spinal cord perfusion pressure experimentally.
\end{abstract}

\begin{abstract}
Methods: In beagle dogs $(\mathrm{n}=8)$, the thoracoabdominal aorta and segmental arteries L1-L7 were exposed, and a temporary bypass was created for distal perfusion. Next, a laser blood flow meter was placed on the spinal dura mater in the L5 region to measure the spinal cord blood flow. The following were measured simultaneously when the direct blood supply from segmental arteries L2-L7 to the spinal cord was stopped: mean systemic blood pressure, spinal cord perfusion pressure (blood pressure within the aortic clamp site), and spinal cord blood flow supplied via the collateral network. These variables were then investigated for evidence of correlations.
\end{abstract}

\begin{abstract}
Results: Positive correlations were observed between mean systemic blood pressure and spinal cord blood flow during interruption of segmental artery flow both with $(r=0.844, P<.01)$ and without $(r=0.834, P<.01)$ distal aortic perfusion. In addition, we observed significant correlations between spinal cord perfusion pressure and spinal cord blood flow with and without distal perfusion $(r=0.803, P<.001$ and $r=0.832, P<.01$, respectively), and between mean systemic blood pressure and spinal cord perfusion pressure with and without distal perfusion ( $r=0.898, P<.001$ and $r=0.837, P<.001$, respectively). The spinal cord was perfused from the collateral network from outside the interrupted segmental arteries, and high systemic blood pressure ( $~ 1.33$-fold higher) was needed to obtain the preclamping spinal cord blood flow, whereas 1.68 -fold higher systemic blood pressure was needed when distal perfusion was halted.
\end{abstract}

Conclusions: Spinal cord blood flow is positively correlated with mean systemic blood pressure and spinal cord perfusion pressure under spinal cord ischemia caused by clamping a wide range of segmental arteries. In open and endovascular thoracic and thoracoabdominal surgery, elevating mean systemic blood pressure is a simple and effective means of increasing spinal cord blood flow, and measuring spinal cord perfusion pressure seems to be useful for monitoring perioperative spinal cord blood flow. (J Thorac Cardiovasc Surg 2015;149:360-6)

See related commentary on pages 366-8.

The complication of greatest concern in thoracoabdominal surgery is spinal cord injury (SCI) from sacrifice of the segmental arteries (SAs), which serve a crucial role in supplying blood flow to the spinal cord. Various methods

From the Department of Thoracic and Cardiovascular Surgery, Graduate School of Medicine, University of the Ryukyus, Okinawa, Japan.

Disclosures: Authors have nothing to disclose with regard to commercial support.

Received for publication May 25, 2014; revisions received Sept 11, 2014; accepted for publication Sept 14, 2014

Address for reprints: Yuya Kise, MD, Department of Thoracic and Cardiovascular Surgery, Graduate School of Medicine, University of the Ryukyus, 207 Uehara, Nakagamigun, Nishihara, Okinawa 903-0215, Japan (E-mail: yuya1022@med. u-ryukyu.ac.jp).

$0022-5223 / \$ 36.00$

Copyright (c) 2015 by The American Association for Thoracic Surgery

http://dx.doi.org/10.1016/j.jtcvs.2014.09.121 are used to avoid this (monitoring motor evoked potential, determining the origin and revascularization of the Adamkiewicz artery [AKA], perfusing the distal aorta, draining cerebrospinal fluid, cooling the spinal cord, and administering certain drugs), but a definitive method has yet to be established. ${ }^{1}$

Recent research on the blood supply to the spinal cord has reported that there is a rich network of spinal cord blood vessels and tissue around the spinal cord that plays an important role in spinal cord circulation. ${ }^{2}$ In actual clinical practice, increasing systemic blood pressure is useful as a means of increasing the blood supply from the collateral network. However, it is not possible to measure spinal cord blood flow (SCBF) in clinical settings, and there are few reports showing a correlation between systemic blood pressure and SCBF.

In this experiment, SCBF was measured directly via the collateral network while blood flow from several SAs, 

Abbreviations and Acronyms
AKA = Adamkiewicz artery
ASA $=$ anterior spinal artery
CSFP $=$ cerebrospinal fluid pressure
$\mathrm{mDAP}=$ mean distal arterial pressure
mPAP $=$ mean proximal arterial pressure
$\mathrm{mSBP}=$ mean systemic blood pressure
SA $=$ segmental artery
SAP $=$ segmental arterial pressure
SCBF $=$ spinal cord blood flow
SCI $=$ spinal cord injury
$\mathrm{SCPP}=$ spinal cord perfusion pressure

including the AKA, was interrupted, and the correlation between SCBF and mean systemic blood pressure (mSBP) was clarified. Spinal cord perfusion pressure (SCPP), which is sometimes used to monitor SCBF perioperatively, was also measured, and its correlations with SCBF and $\mathrm{mSBP}$ were examined.

In addition, changes in SCBF with and without distal perfusion were measured, and the degree to which distal perfusion affects SCBF via the collateral network was investigated. Finally, the mSBP conditions to maintain SCBF before interruption of the SAs were investigated. The aim of this study was to investigate spinal cord blood supply through a collateral network by directly measuring SCBF and SCPP experimentally.

\section{MATERIALS AND METHODS Animals}

Animal care and all procedures were performed in compliance with the Guide for Care of Laboratory Animals. This study was approved by the Research Committee for Laboratory Animal Science at the University of the Ryukyus. Experiments were performed on 8 female beagle dogs weighing 7.5 to $10.0 \mathrm{~kg}$.

\footnotetext{
Anesthesia

The dogs were sedated by intramuscular injection of $3 \mathrm{mg} / \mathrm{kg}$ ketamine hydrochloride and $0.2 \mathrm{mg}$ atropine sulfate. They were then intubated endotracheally and ventilated mechanically. One intravenous line was inserted into an anterior limb vein. They were placed in the prone position on an operating table under intravenous anesthesia with propofol $(0.3 \mathrm{mg} / \mathrm{kg} / \mathrm{min})$ and ketamine hydrochloride (0.05 mg/kg/min).

During the surgical procedure, the mean arterial blood pressure was maintained between 70 and $100 \mathrm{~mm} \mathrm{Hg}$ by controlling the propofol concentration and fluid infusion. Body temperature was monitored with a rectal temperature probe and maintained between $36^{\circ} \mathrm{C}$ and $37^{\circ} \mathrm{C}$ using a heating pad and blanket. Arterial blood gases were measured (i-STAT1; FUSO Pharmaceutical, Ltd, Osaka, Japan) at 60-minute intervals. Metabolic and respiratory acid-base balance was confirmed to maintain $\mathrm{pH}$ between 7.35 and 7.45, arterial oxygen tension was maintained at greater than $100 \mathrm{~mm} \mathrm{Hg}$, and arterial carbon dioxide tension was maintained between 35 and $45 \mathrm{~mm} \mathrm{Hg}$ by adjusting respiratory volume and rate.
}

\section{Surgical Procedure}

Surgery was performed by a single cardiovascular surgeon to exclude effects on measurements related to surgeon expertise.

First, an L4 laminectomy was performed, and the dorsal aspect of the dura mater, measuring $1.5 \times 1.5 \mathrm{~cm}$, over the spinal cord was exposed. Second, the animals were placed in the right decubitus position. The chest was opened through a thoracotomy in the ninth left intercostal space. The abdominal aorta was exposed through a left flank incision and exfoliated from the descending aorta (Th11) to the trifurcation, with careful exposure of the L2-L7 SAs. After heparin was administered intravenously at $200 \mathrm{IU} / \mathrm{kg}$, a temporary descending aorta to left external iliac artery bypass was created. At proximal sites, a $10 \mathrm{~F}$ aortic cannula (Duraflo II; Edwards Lifesciences, Irvine, Calif) was inserted, and at distal sites, an 8-mm woven graft was anastomosed, and these devices were connected.

Then, 22-G cannulae were placed at the left common carotid artery, right femoral artery, and L5 level abdominal aorta to monitor the mean proximal arterial pressure (mPAP), mean distal arterial pressure (mDAP), and segmental arterial pressure (SAP), respectively. The SAP substituted for SCPP while the abdominal aorta was clamped (between L3 to L4 and L6 to L7) (Figure 1). Because the AKA branches from the L4 or L5 SA in dogs, this range of interruption included the AKA..$^{3-5}$

\section{Measurement of Spinal Cord Blood Flow}

By referring to Fujimaki and colleagues' procedure, ${ }^{6}$ SCBF was measured using laser Doppler flowmetry (Omegaflow FLO-N1; Neuroscience, Tokyo, Japan) to assess real-time microcirculatory changes in the spinal cord. The laser probe was placed in contact with the intact dorsal dura mater at the L5 segmental level of the spinal cord, which was at the upper L4 vertebral level and connected to the laser Doppler flow meter. The probe was affixed in a riding position over the spinal cord. Output signals were collected continuously throughout the experiment and averaged every 3 seconds.

\section{Experimental Protocol}

Mean blood pressures (mPAP, mDAP, SAP [SCPP]) and SCBF were measured at the same time under the following 3 conditions (Figure 2): condition 1, no aortic clamp and no SA clamp (control group); condition 2, aortic clamp (between L3 to L4 and L6 to L7), and L2, L3, and L7 SA clamps (L2-L7 SA flow halted) with distal perfusion; and condition 3, aortic clamp (between L3 to L4 and L6 to L7) and L2, L3, and L7 SA clamps (L2-L7 SA flow halted) with no distal perfusion.

The mean values of each measurement over 3 minutes from the start of measurements were taken as the baseline values. After completing the baseline measurements, condition 1 was created, and continuous administration of $0.5 \mu \mathrm{g} / \mathrm{kg} / \mathrm{min}$ norepinephrine was started. Measurement data for each site were sampled every 15 seconds from the start of the increase in $\mathrm{mSBP}$ (the mPAP measurement value was taken to be $\mathrm{mSBP})$. The continuous administration of norepinephrine was stopped after 5 minutes, and data sampling was performed for approximately 10 minutes as blood pressure naturally decreased and stabilized. The same procedure was continued for conditions 2 and 3. A difference of less than $10 \mathrm{~mm} \mathrm{Hg}$ between mPAP and mDAP was taken to indicate that the distal bypass during the experiment was functioning effectively.

\section{Data Analysis}

All data were recorded by the monitor (PowerLab, ADInstruments, ADInstruments Pty Ltd, Castle Hill, Australia), which allowed constant real-time recording of the arterial blood pressure (mPAP, mDAP, SAP [SCPP]) and SCBF. Data were entered into a database and analyzed using SPSS statistical software (version 21.0J; IBM Corp, Armonk, NY).

Scatter plots of mSBP and SCBF, SAP (SCPP) and SCBF, and mSBP and SAP (SCPP) were created, and levels of correlation were analyzed with Spearman's rank correlation coefficient and regression analysis. A probability of $5 \%$ was considered significant. 

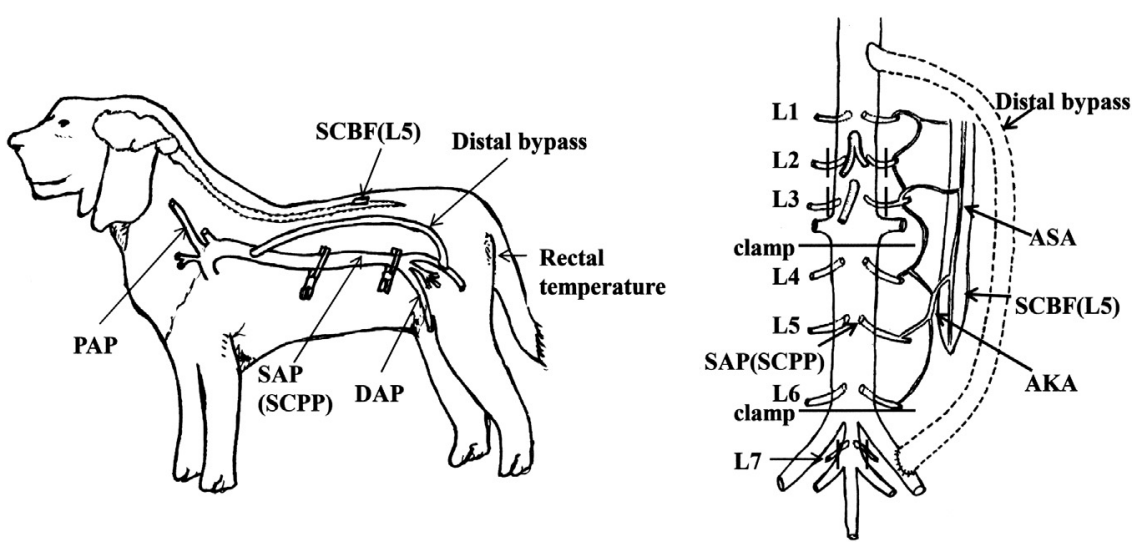

FIGURE 1. Experimental model. $A K A$, Adamkiewicz artery; $A S A$, anterior spinal artery; $D A P$, distal arterial blood pressure; $P A P$, proximal arterial blood pressure; $S A P$, segmental arterial pressure; $S C B F$, spinal cord blood flow; $S C P P$, spinal cord perfusion pressure.

\section{RESULTS}

\section{Condition 1}

When blood flow was maintained from the SAs, SCBF increased mildly relative to the increase in $\mathrm{mSBP}$ (Figure 3, $A$ ). The scattergram of percentage changes revealed that there was a weak positive correlation between $\mathrm{mSBP}$ and $\operatorname{SCBF}\left(r=0.403, r^{2}=0.162, P<.01\right)$ (Figure 3, $\left.B\right)$.

\section{Condition 2}

Under condition 2, inflow from the SAs at L2-L7 was halted with distal perfusion. When inflow from the SAs was stopped, SCBF and SAP (SCPP) decreased, but they increased as $\mathrm{mSBP}$ increased (Figure 4, A).

The scattergram of percentage changes showed that there were positive correlations between $\mathrm{mSBP}$ and SCBF ( $\left.r=0.844, r^{2}=0.711, P<.01\right)$, between SAP (SCPP) and SCBF $\left(r=0.803, r^{2}=0.644, P<.001\right)$, and between $\mathrm{mSBP}$ and SAP (SCPP) $\left(r=0.898, r^{2}=0.806, P<.001\right)$ (Figure 4, $B$ - $D$ ).

\section{Condition 3}

After condition 2, distal perfusion was halted. When the distal bypass was clamped, SCBF and SAP (SCPP) decreased further, but they then increased as mSBP increased (Figure 5, A). The scattergram of percentage changes showed that there were positive correlations between $\mathrm{mSBP}$ and $\mathrm{SCBF}\left(r=0.834, r^{2}=0.695\right.$, $P<.01)$, between SAP (SCPP) and SCBF $(r=0.832$, $\left.r^{2}=0.692, P<.01\right)$, and between $\mathrm{mSBP}$ and SAP (SCPP) $\left(r=0.837, r^{2}=0.700, P<.001\right)$ (Figure $\left.5, B-D\right)$.

\section{DISCUSSION}

SCI (paraparesis and paraplegia) caused by interruption of the blood supply to the spinal cord from SAs is one of the greatest concerns in thoracic and thoracoabdominal aortic aneurysm surgery. The AKA is a particularly important blood supply route to the anterior spinal artery (ASA), but in extensive aortic aneurysm surgeries, several pairs of SAs including the AKA are sacrificed. This makes
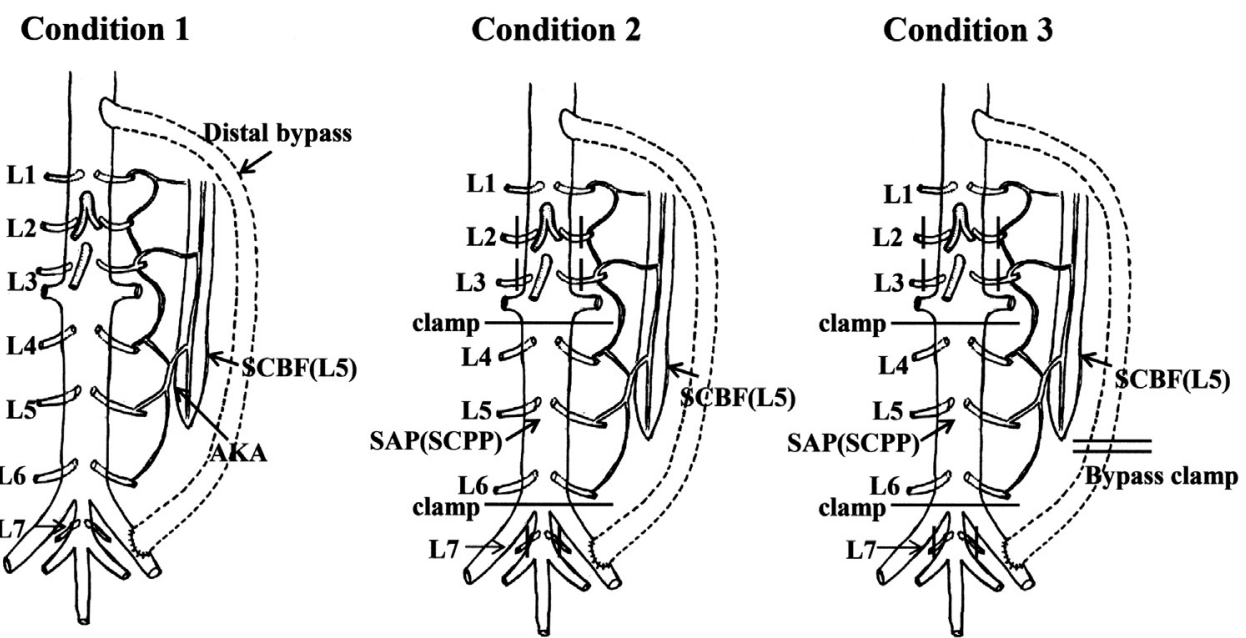

FIGURE 2. Experimental protocol. Condition 1, no aortic clamp and no SA clamp (control group); condition 2, L2-L7 SA flow halted with distal perfusion; condition 3, L2-L7 SA flow halted with no distal perfusion. AKA, Adamkiewicz artery; SAP, segmental arterial pressure; $S C B F$, spinal cord blood flow; $S C P P$, spinal cord perfusion pressure. 


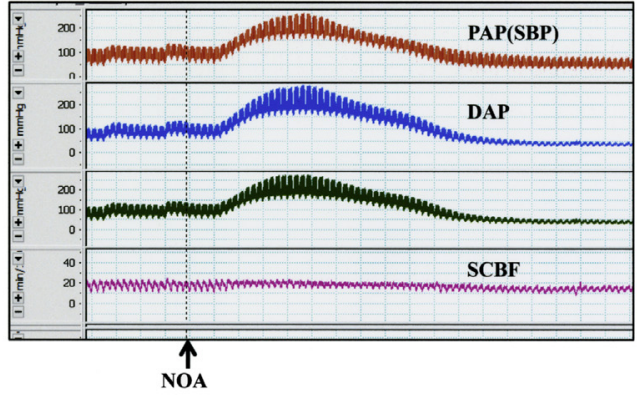

A

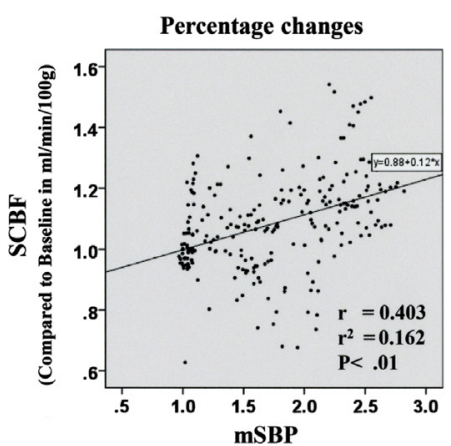

B

(Compared to Baseline in mmHg)

FIGURE 3. Condition 1, no aortic clamp and no SA clamp (control group). A, Laboratory chart shows the real-time record of systemic blood pressure, distal arterial blood pressure, and SCBF. SCBF increases mildly relative to the increase in mSBP. B, Scattergram of percentage changes shows a weak positive correlation between $\mathrm{mSBP}$ and SCBF. $D A P$, Distal aortic blood pressure; $m S B P$, mean systemic blood pressure; NOA, norepinephrine; $P A P$, proximal arterial blood pressure; $S B P$, systemic blood pressure; $S C B F$, spinal cord blood flow.

preservation of perioperative SCBF an issue. At such times, SCBF depends on flow from the collateral network, but experimental studies in recent years have shown that the collateral network to the spinal cord serves an important role in preserving $\mathrm{SCBF}^{2,7-10}$

In actual clinical practice, SCBF decreases with sacrifice of the SAs, but SCBF from the collateral network is increased by augmenting the systemic blood pressure with the use of volume load or vasopressors such as phenylephrine and norepinephrine. This has been shown to result in effective reduction of SCI. ${ }^{11-14}$

This suggests a positive correlation between SCBF and systemic blood pressure, but direct measurement of SCBF is difficult in actual clinical settings. Vascularization of
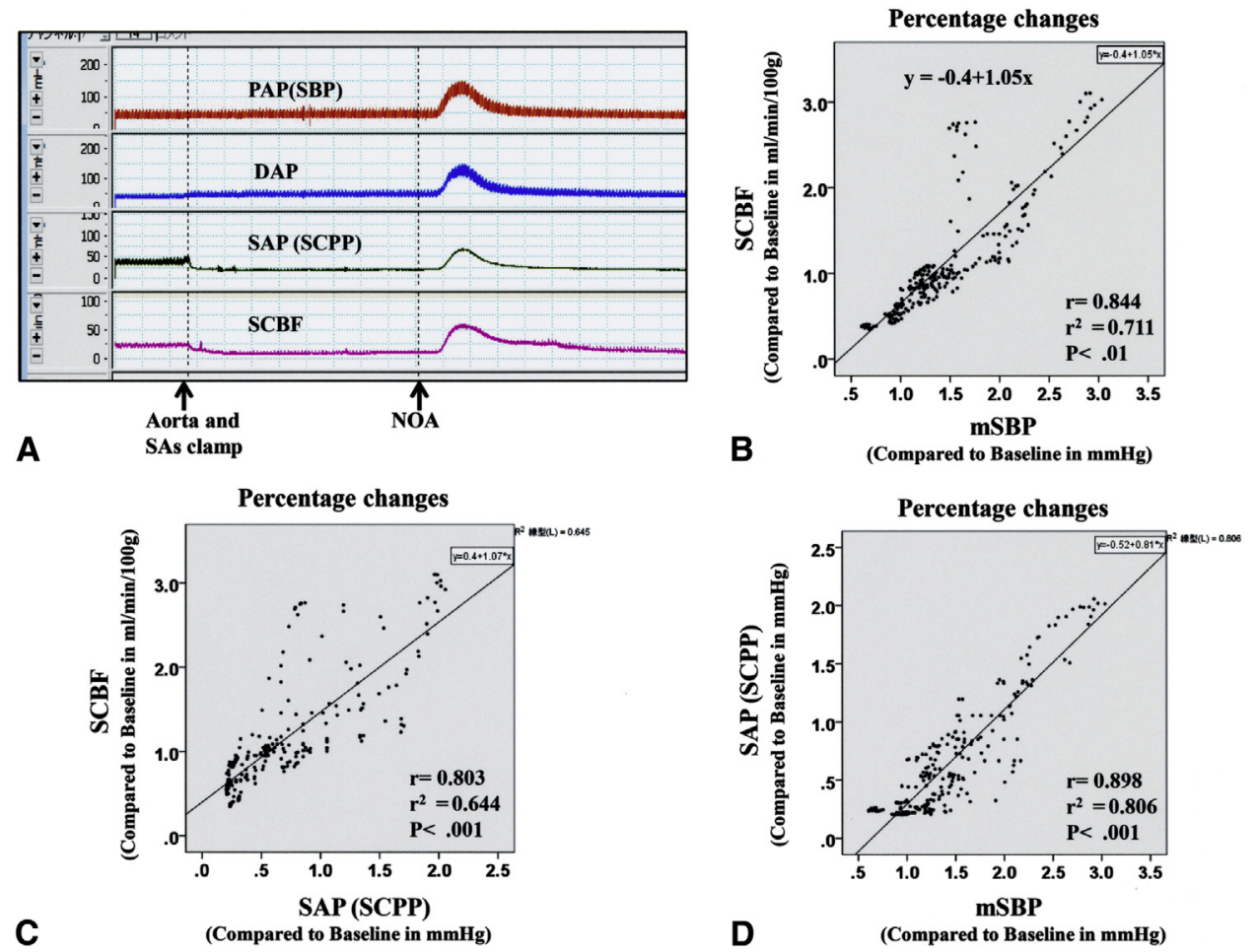

FIGURE 4. Condition 2, aortic clamp (between L3 to L4 and L6 to L7) and L2, L3, and L7 SA clamps (L2-L7 SA flow halted) with distal perfusion. A, When inflow from the SAs is stopped, SCBF and SAP (SCPP) decrease; however, they increase as systemic blood pressure increases. B, Scattergram of percentage changes shows a positive correlation between mSBP and SCBF. C, Positive correlation is seen between SAP (SCPP) and SCBF. D, Positive correlation is seen between mSBP and SAP (SCPP). DAP, Distal arterial blood pressure; $m S B P$, mean systemic blood pressure; NOA, norepinephrine; $P A P$, proximal arterial blood pressure; $S A$, segmental artery; $S A P$, segmental arterial pressure; $S B P$, systemic blood pressure; $S C B F$, spinal cord blood flow; $S C P P$, spinal cord perfusion pressure. 


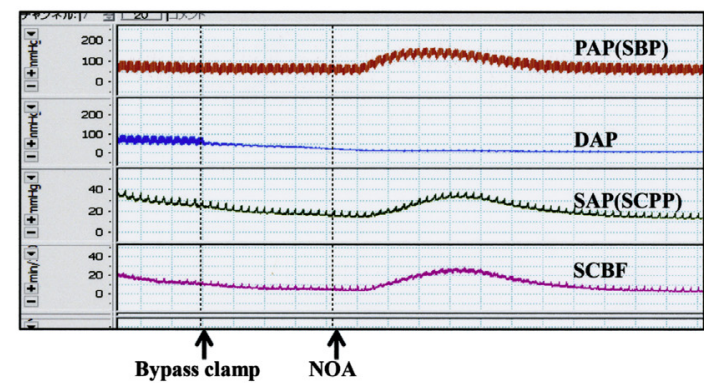

A

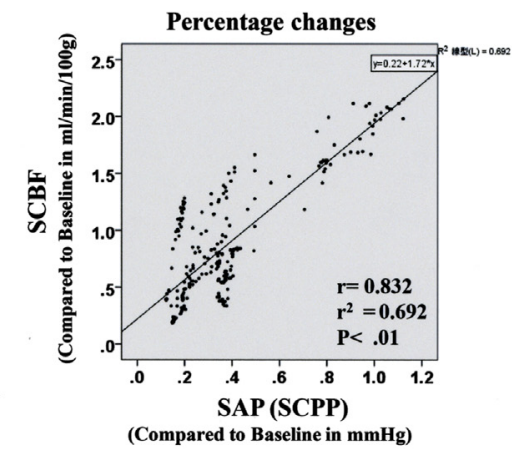

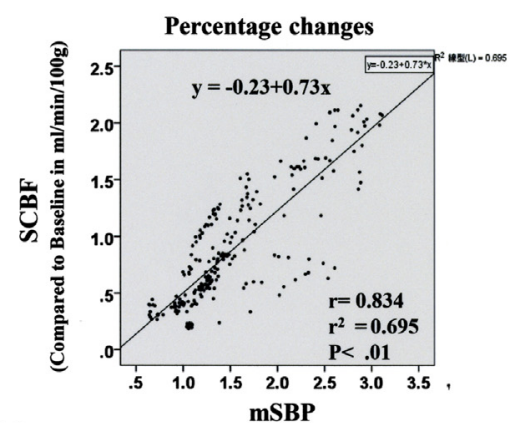

B

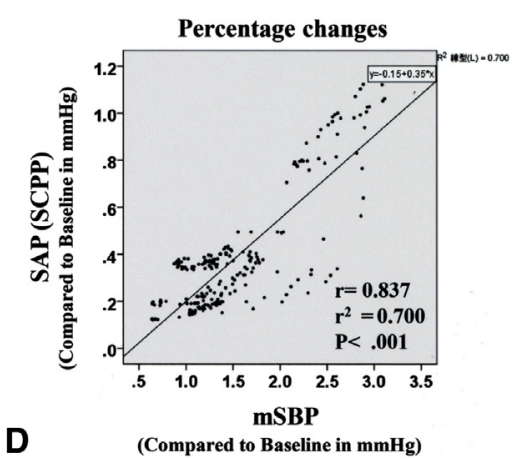

FIGURE 5. Condition 3, aortic clamp (between L3 to L4 and L6 to L7) and L2, L3, and L7 SA clamps (L2-L7 SA flow halted) with no distal perfusion. A, When the distal bypass is clamped, SCBF and SAP (SCPP) decrease further; however, they then increase as systemic blood pressure increases. B, Scattergram of percentage changes shows a positive correlation between $\mathrm{mSBP}$ and SCBF. C, Positive correlation is seen between SAP (SCPP) and SCBF. D, Positive correlation is seen between mSBP and SAP (SCPP). DAP, Distal arterial blood pressure; $m S B P$, mean systemic blood pressure; $N O A$, norepinephrine; $P A P$, proximal arterial blood pressure; $S A P$, segmental arterial pressure; $S B P$, systemic blood pressure; $S C B F$, spinal cord blood flow; $S C P P$, spinal cord perfusion pressure.

the spinal cord of dogs is similar to that of human beings, and dogs have a rich anastomotic network of spinal cord vascularization, particularly at the conus medullaris, as do human beings. ${ }^{3,5}$ In this experimental study, 6 pairs of SAs including the AKA were interrupted, and the $\mathrm{mSBP}$ and SCBF were directly measured using a laser blood flow meter that can measure SCBF in real time..$^{15,16}$ The correlation between them was then examined.

A correlation between mSBP and SCBF in a simple aortic crossclamping model in previous experimental research has been reported, ${ }^{17,18}$ but in the present study, SAs were interrupted, and distal perfusion was maintained by a distal bypass. This has been shown to continuously maintain distal perfusion intraoperatively and postoperatively in endovascular aortic repair, whereas distal perfusion with left heart bypass or femoral-femoral partial bypass for assisted circulation has been shown to be effective in protecting the spinal cord in open thoracoabdominal surgery. ${ }^{19-21}$ An experimental model to simulate this was devised. The clear decrease in SCBF with distal bypass interruption in this experiment showed that distal perfusion contributed significantly to SCBF via the collateral network.

The results of this experiment showed a highly positive correlation between the rates of increase in $\mathrm{mSBP}$ and
SCBF when 6 SAs including the AKA were interrupted. Under distal perfusion, a simple linear regression line showed that mSBP augmentation of 1.33-fold is needed after SA interruption to maintain the blood flow from before the interruption, whereas mSBP augmentation of 1.68-fold is needed with nondistal perfusion. This is the first report to experimentally measure and quantify the degree to which blood pressure needs to be increased to maintain SCBF. It is also significant in that it simultaneously shows the degree to which distal perfusion contributes to spinal cord circulation.

At the same time, a weak positive correlation was seen between the increases in $\mathrm{mSBP}$ and SCBF in the control group that did not have interrupted $\mathrm{SAs}$ in this experiment. SCBF did not change with an $\mathrm{mSBP}$ of 50 to $135 \mathrm{~mm} \mathrm{Hg}$ in a monkey experiment, ${ }^{22} \mathrm{mSBP}$ of 40 to $100 \mathrm{~mm} \mathrm{Hg}$ in a lamb experiment, ${ }^{23}$ or mSBP of 80 to $160 \mathrm{~mm} \mathrm{Hg}$ in a cat experiment. ${ }^{24}$ These studies demonstrated the blood pressure range in which autoregulation acts in the SCBF experimentally. The paravertebral sympathetic ganglia also have been reported to have a specific role in autoregulation of the SCBF. ${ }^{24}$ In this study, spinal cord circulation collapsed with extensive SA interruption, autoregulation was not seen, and a positive correlation was seen between the 
increase in $\mathrm{mSBP}$ and the increase in SCBF from the collateral network.

It is thought that SCI can be protected by maintaining the SCPP at 50 to $60 \mathrm{~mm} \mathrm{Hg}$ or greater. ${ }^{25} \mathrm{Etz}$ and colleagues ${ }^{26-28}$ placed a blood pressure monitoring catheter for several days in the distal end of the ligated SA at the time of thoracoabdominal surgery to measure SCPP both intraoperatively and postoperatively, and they stated that this was effective in managing spinal cord circulation. Because it is difficult to place a catheter in the fine SAs of dogs, SCPP was substituted with placement of blood pressure monitoring catheters in the area of the aortic interruption (L4-L6).

The results of this experiment showed a high positive correlation between SAP (SCPP) and SCBF, and monitoring of SAP (SCPP) after interruption of SAs is thought to be useful in appropriately maintaining spinal cord circulation. In addition, systemic blood pressure was found to be positively correlated with SAP (SCPP).

Blood supply from the collateral network was increased by increasing the mSBP to deal with the decrease in SCBF from sacrificing the SAs. In several experimental models, nerve damage after extensive SA sacrifice was shown to begin 1 to 5 hours postoperatively, ${ }^{26,29}$ and maintaining a somewhat high blood pressure with hemodynamic manipulation for 24 to 72 hours until the collateral network develops ${ }^{8,14,29}$ was demonstrated experimentally to be important as a means of preventing SCI.

\section{Study Limitations}

The erector spinae muscles are a source of some of the collateral network blood supply, but slight damage to these muscles could not be avoided during laminectomy and exposure of L5 dura mater. In addition, SCBF was measured on the dorsal side of the spinal cord, and because of the properties of the laser blood flow meter, tissue blood flow is reflected to a depth of only approximately $1 \mathrm{~mm}$. Consequently, blood flow in the ASA region of the ventral spinal cord could not be measured directly. However, because there is communication between the posterospinal artery and the anterospinal artery via the epidural arcade and pial arterial plexus, increased SCBF on the dorsal side of the spinal cord is thought to reflect increased SCBF in the ASA region. ${ }^{1,2,8,9}$

SCPP is accurately expressed with SAP-cerebrospinal fluid pressure (CSFP). It would have been preferable to measure CSFP and show SAP-CSFP as SCPP in this experiment as well, but measuring CSFP was difficult because of the experimental procedures. Therefore, it is thought that the SCPP shown in this experiment should be used as an approximation of the actual SCPP.

Norepinephrine was used to increase the blood pressure because it is a drug generally used in cardiovascular surgery, but the pharmacologic actions of norepinephrine on spinal cord vessels are not clear, and the effect of this will need to be clarified in future research.

This study did not show a relationship between changes in SCBF and neurologic or histologic changes. Appropriate simultaneous monitoring of transcranial motor evoked potentials is thought to be useful in evaluating spinal cord function. ${ }^{30,31}$

\section{CONCLUSIONS}

SCBF is positively correlated with $\mathrm{mSBP}$ and SCPP under spinal cord ischemia caused by clamping a wide range of segmental arteries. In open and endovascular thoracic and thoracoabdominal surgery, elevating $\mathrm{mSBP}$ is a simple and effective means of increasing SCBF.

The authors thank Tetuji Chinen, Chisato Kamiya, Yuichi Totuka, Takahumi Kozaki, and Yuji Kaneshiro for technical assistance.

\section{References}

1. Martirosyan NL, Feuerstein JS, Theodore N, Cavalcanti DD, Spetzler RF, Preul MC. Blood supply and vascular reactivity of the spinal cord under normal and pathological conditions. J Neurosurg Spine. 2011;15:238-51.

2. Etz CD, Kari FA, Mueller CS, Silovitz D, Brenner RM, Lin HM, et al. The collateral network concept: a reassessment of the anatomy of spinal cord perfusion. $J$ Thorac Cardiovasc Surg. 2011;141:1020-8.

3. Sindou M, Chignier E, Mazoyer JF, Pialat J, Fischer G, Descotes J. Revascularization of the spinal cord by micro-anastomoses in dogs. Surg Neurol. 1979; 12:492-5.

4. Kato S, Kawahara N, Tomita K, Murakami H, Demura S, Fujimaki Y. Effects on spinal cord blood flow and neurologic function secondary to interruption of bilateral segmental arteries which supply the artery of Adamkiewicz: an experimental study using a dog model. Spine. 2008;33: 1533-41.

5. Doppman JL, Ramsey R. Selective arteriography of the lumbar spinal cord in dogs. Neuroradiology. 1971;3:64-7.

6. Fujimaki Y, Kawahara N, Tomita K, Murakami H, Ueda Y. How many ligations of bilateral segmental arteries cause ischemic spinal cord dysfunction? An experimental study using a dog model. Spine. 2006;31:E781-9.

7. Uezu T, Koja K, Kuniyoshi Y, Miyagi K, Shimoji M, Arakaki K, et al. Blood distribution to the anterior spinal artery from each segment of intercostal and lumbar arteries. J Cardiovasc Surg. 2003;44:637-45.

8. Geisbusch S, Schray D, Bischoff MS, Lin HM, Griepp RB, Di Luozzo G. Imaging of vascular remodeling after simulated thoracoabdominal aneurysm repair. J Thorac Cardiovasc Surg. 2012;144:1471-8.

9. Etz CD, Kari FA, Mueller CS, Brenner RM, Lin HM, Griepp RB. The collateral network concept: remodeling of the arterial collateral network after experimental segmental artery sacrifice. J Thorac Cardiovasc Surg. 2011;141: 1029-36.

10. Griepp RB, Griepp EB. Spinal cord perfusion and protection during descending thoracic and thoracoabdominal aortic surgery: the collateral network concept Ann Thorac Surg. 2007;83:S865-9; discussion S90-2.

11. Chiesa R, Melissano G, Marrocco-Trischitta MM, Civilini E, Setacci F. Spinal cord ischemia after elective stent-graft repair of the thoracic aorta. J Vasc Surg. 2005;42:11-7.

12. Kawanishi Y, Okada K, Matsumori M, Tanaka H, Yamashita T, Nakagiri K, et al Influence of perioperative hemodynamics on spinal cord ischemia in thoracoabdominal aortic repair. Ann Thorac Surg. 2007;84:488-92.

13. Hnath JC, Mehta M, Taggert JB, Sternbach Y, Roddy SP, Kreienberg PB, et al Strategies to improve spinal cord ischemia in endovascular thoracic aortic repair: outcomes of a prospective cerebrospinal fluid drainage protocol. J Vasc Surg. 2008;48:836-40. 
14. Ullery BW, Cheung AT, Fairman RM, Jackson BM, Woo EY, Bavaria J, et al. Risk factors, outcomes, and clinical manifestations of spinal cord ischemia following thoracic endovascular aortic repair. J Vasc Surg. 2011;54:677-84.

15. Lindsberg PJ, O'Neill JT, Paakkari IA, Hallenbeck JM, Feuerstein G. Validation of laser-Doppler flowmetry in measurement of spinal cord blood flow. Am J Physiol. 1989;257(2 Pt 2):H674-80.

16. Lindsberg PJ, Jacobs TP, Frerichs KU, Hallenbeck JM, Feuerstein GZ Laser-Doppler flowmetry in monitoring regulation of rapid microcirculatory changes in spinal cord. Am J Physiol. 1992;263(1 Pt 2):H285-92.

17. Taira Y, Marsala M. Effect of proximal arterial perfusion pressure on function, spinal cord blood flow, and histopathologic changes after increasing intervals of aortic occlusion in the rat. Stroke. 1996;27:1850-8.

18. Izumi S, Okada K, Hasegawa T, Omura A, Munakata H, Matsumori M, et al. Augmentation of systemic blood pressure during spinal cord ischemia to prevent postoperative paraplegia after aortic surgery in a rabbit model. J Thorac Cardiovasc Surg. 2010;139:1261-8

19. Laschinger JC, Cunningham JN Jr, Nathan IM, Knopp EA, Cooper MM, Spencer FC. Experimental and clinical assessment of the adequacy of partial bypass in maintenance of spinal cord blood flow during operations on the thoracic aorta. Ann Thorac Surg. 1983;36:417-26.

20. Strauch JT, Spielvogel D, Lauten A, Zhang N, Shiang H, Weisz D, et al. Importance of extrasegmental vessels for spinal cord blood supply in a chronic porcine model. Eur J Cardiothorac Surg. 2003;24:817-24.

21. Safi HJ, Miller CC III, Huynh TT, Estrera AL, Porat EE, Winnerkvist AN, et al. Distal aortic perfusion and cerebrospinal fluid drainage for thoracoabdominal and descending thoracic aortic repair: ten years of organ protection. Ann Surg. 2003;238:372-81.

22. Kobrine AI, Evans DE, Rizzoli HV. The role of the sympathetic nervous system in spinal cord autoregulation. Acta Neurol Scand Suppl. 1977;64:54-5.
23. Hitchon PW, Lobosky JM, Yamada T, Johnson G, Girton RA. Effect of hemorrhagic shock upon spinal cord blood flow and evoked potentials. Neurosurgery. 1987;21:849-57.

24. Young W, DeCrescito V, Tomasula JJ. Effect of sympathectomy on spinal blood flow autoregulation and posttraumatic ischemia. J Neurosurg. 1982;56:706-10.

25. Kazama S, Masaki Y, Maruyama S, Ishihara A. Effect of altering cerebrospinal fluid pressure on spinal cord blood flow. Ann Thorac Surg. 1994 58:112-5.

26. Etz CD, Homann TM, Plestis KA, Zhang N, Luehr M, Weisz DJ, et al. Spinal cord perfusion after extensive segmental artery sacrifice: can paraplegia be prevented? Eur J Cardiothorac Surg. 2007:31:643-8.

27. Etz CD, Di Luozzo G, Zoli S, Lazala R, Plestis KA, Bodian CA, et al. Direct spinal cord perfusion pressure monitoring in extensive distal aortic aneurysm repair. Ann Thorac Surg. 2009;87:1764-74.

28. Etz CD, Zoli S, Bischoff MS, Bodian C, Di Luozzo G, Griepp RB. Measuring the collateral network pressure to minimize paraplegia risk in thoracoabdominal aneurysm resection. J Thorac Cardiovasc Surg. 2010;140(6 Suppl):S125-30; discussion S42-6.

29. Etz CD, Homann TM, Luehr M, Kari FA, Weisz DJ, Kleinman G, et al. Spinal cord blood flow and ischemic injury after experimental sacrifice of thoracic and abdominal segmental arteries. Eur J Cardiothorac Surg. 2008;33: 1030-8.

30. Lips J, de Haan P, de Jager SW, Vanicky I, Jacobs MJ, Kalkman CJ. The role of transcranial motor evoked potentials in predicting neurologic and histopathologic outcome after experimental spinal cord ischemia. Anesthesiology. 2002;97: 183-91.

31. Weigang E, Hartert M, Siegenthaler MP, Pitzer-Hartert K, Luehr M, Sircar R, et al. Neurophysiological monitoring during thoracoabdominal aortic endovascular stent graft implantation. Eur J Cardiothorac Surg. 2006;29:392-6.

\title{
EDITORIAL COMMENTARY
}

\section{Preventing spinal cord injury during thoracic aortic surgery: Simpler than we thought?}

\author{
Hiroo Takayama, MD, PhD, and Michael A. Borger, MD, PhD
}

See related article on pages $360-6$.

Although more than 2 decades has passed since the groundbreaking clinical work by Stanley Crawford and his colleagues, spinal cord injury and subsequent paraplegia remain the most dreaded complication after thoracoabdominal aortic aneurysm (TAAA) repair. In a recent review article studying 38,491 patients pooled from multiple

From the Division of Cardiac, Thoracic and Vascular Surgery, Columbia University Medical Center, New York, NY.

Disclosures: Authors have nothing to disclose with regard to commercial support.

Received for publication Oct 25, 2014; accepted for publication Oct 27, 2014; available ahead of print Nov 25, 2014

Address for reprints: Michael A. Borger, MD, PhD, Division of Cardiac, Thoracic, and Vascular Surgery, Columbia University Medical Center, 177 Fort Washington Ave, MHB 7GN-435, New York, NY 10032 (E-mail: mb3851@cumc.columbia. edu).

J Thorac Cardiovasc Surg 2015;149:366-8

0022-5223/\$36.00

Copyright (c) 2015 by The American Association for Thoracic Surgery

http://dx.doi.org/10.1016/j.jtcvs.2014.10.106 studies, the overall incidence of paraplegia or paraparesis was $7.1 \%$, with a range of $0 \%$ to $32 \%$. ${ }^{1}$ The incidence decreased from $9.0 \%$ before 2000 to $7.0 \%$ between 2001 and 2007, and then to 5.9\% between 2008 and 2013 . Although the incidence of paraplegia appears to be decreasing, this complication remains a significant problem because of its grave impact on patient quality of life. Endovascular aneurysm repair is being performed more frequently in patients with aortic disease, but even these procedures are known to be associated with spinal cord injury. We must therefore continue to find ways to decrease the occurrence of this devastating complication in patients with TAAA.

The cause of spinal cord injury during TAAA repair is multifactorial. A multitude of preventative and therapeutic approaches have therefore been developed, including distal aortic perfusion, maintenance of mean systolic arterial pressure, cerebrospinal fluid drainage, hypothermia, epidural cooling, selective spinal cord perfusion, sequential aortic crossclamping, reimplantation of intercostal arteries, 